\title{
Room-Temperature Low-Threshold Lasing from Monolithically Integrated Nanostructured Porous Silicon Hybrid Microcavities
}

\author{
Valentina Robbiano, ${ }^{\dagger}$ Giuseppe M. Paternò ${ }^{\ddagger}{ }^{\dagger}$ Antonino A. La Mattina, ${ }^{\dagger}$ Silvia G. Motti, \\ Guglielmo Lanzani, ${ }^{\frac{1}{+}, \S}$ Francesco Scotognella, ${ }^{+, \S}$ and Giuseppe Barillaro*, ${ }^{*} \dagger$
}

${ }^{\dagger}$ Dipartimento di Ingegneria dell’Informazione, Università di Pisa, Via G. Caruso 16, 56122 Pisa, Italy

${ }^{\ddagger}$ Center for Nano Science and Technology@PoliMi, Istituto Italiano di Tecnologia, Via Giovanni Pascoli, 70/3, 20133 Milano, Italy

${ }^{\S}$ Dipartimento di Fisica, Politecnico di Milano, Piazza Leonardo da Vinci 32, 20133 Milano, Italy

Supporting Information

\begin{abstract}
Silicon photonics would strongly benefit from monolithically integrated low-threshold silicon-based laser operating at room temperature, representing today the main challenge toward low-cost and power-efficient electronic-photonic integrated circuits. Here we demonstrate low-threshold lasing from fully transparent nanostructured porous silicon (PSi) monolithic microcavities (MCs) infiltrated with a polyfluorene derivative, namely, poly(9,9-di-n-octylfluorenyl-2,7-diyl) (PFO). The PFOinfiltrated PSiMCs support single-mode blue lasing at the resonance wavelength of $466 \mathrm{~nm}$, with a line width of $\sim 1.3 \mathrm{~nm}$ and lasing threshold of $5 \mathrm{~nJ}\left(15 \mu \mathrm{J} / \mathrm{cm}^{2}\right)$, a value that is at the state of the art of PFO lasers. Furthermore, time-resolved photoluminescence shows a significant shortening ( $\sim 57 \%)$ of PFO

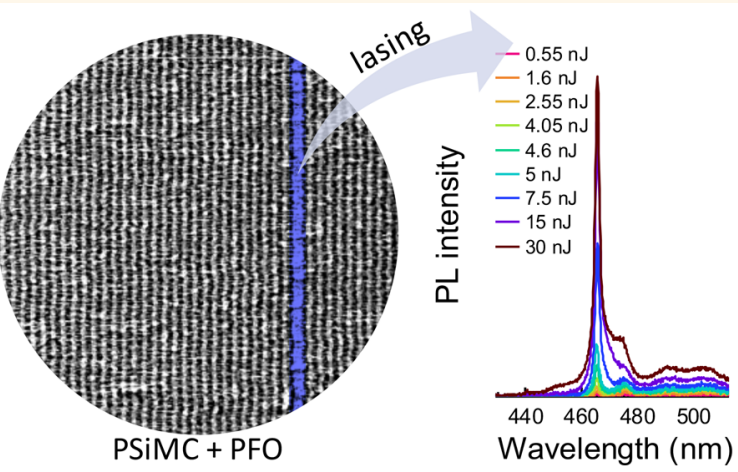
emission lifetime in the PSiMCs, with respect to nonresonant PSi reference structures, confirming a dramatic variation of the radiative decay rate due to a Purcell effect. Our results, given also that blue lasing is a worst case for silicon photonics, are highly appealing for the development of low-cost, lowthreshold silicon-based lasers with wavelengths tunable from visible to the near-infrared region by simple infiltration of suitable emitting polymers in monolithically integrated nanostructured PSiMCs.
\end{abstract}

KEYWORDS: silicon lasing, organic laser, porous silicon, resonant cavity, organic semiconductor, polyfluorene

ince the first report of room-temperature photoluminescence from nanostructured porous silicon $(\mathrm{PSi}){ }^{1}$, scientists have fantasized about PSi-based lasers, ${ }^{2}$ enabling the realization of integrated silicon photonic circuits. ${ }^{3}$ Although some trials on the fabrication of a $\mathrm{Si}$ nanocrystalbased laser have been attempted after the discovery of Si nanocrystal optical gain, a PSi-based laser has not been reported yet. ${ }^{4}$ In the following two decades, PSi has been more successfully used in optical biosensing ${ }^{5,6}$ and nanomedicine, 7,8 owing to its vast surface-to-volume ratio (up to $1000 \mathrm{~m}^{2} / \mathrm{cm}^{3}$ ) and unusually long emission lifetime $(5-13 \mu \mathrm{s})$.

Porous silicon is easily prepared via controlled electrochemical dissolution of crystalline silicon at room temperature. $^{10}$ Interestingly, the mass of silicon removed for unit volume (i.e., the porosity) is proportional to the etching current density value. This provides a powerful tool to finely set PSi porosity value and profile and, in turn, to accurately tune the refractive index of PSi by simply controlling the current density value and waveform over time. ${ }^{11-13}$ In this way, a variety of mesoporous photonic structures (e.g., Bragg reflectors (DBRs) ${ }^{14,15}$ resonant microcavities (MCs), ${ }^{16,17}$ rugate filters, ${ }^{18,19}$ waveguides ${ }^{20}$ ) operating in both the visible and nearinfrared regions were prepared in PSi through controlled modulation of the refractive index.

In the past few years, leveraging a cheap and robust material preparation technique coupled with a high flexibility and high quality in optical structure fabrication, PSi has gained renewed interest in integrated optics and photonics. Gradient refractive index (GRIN) optical elements, ${ }^{21}$ either $2 \mathrm{D}$ or $3 \mathrm{D}$, capable of

Received: February 2, 2018

Accepted: $\quad$ May 4, 2018

Published: May 4, 2018 
(a)
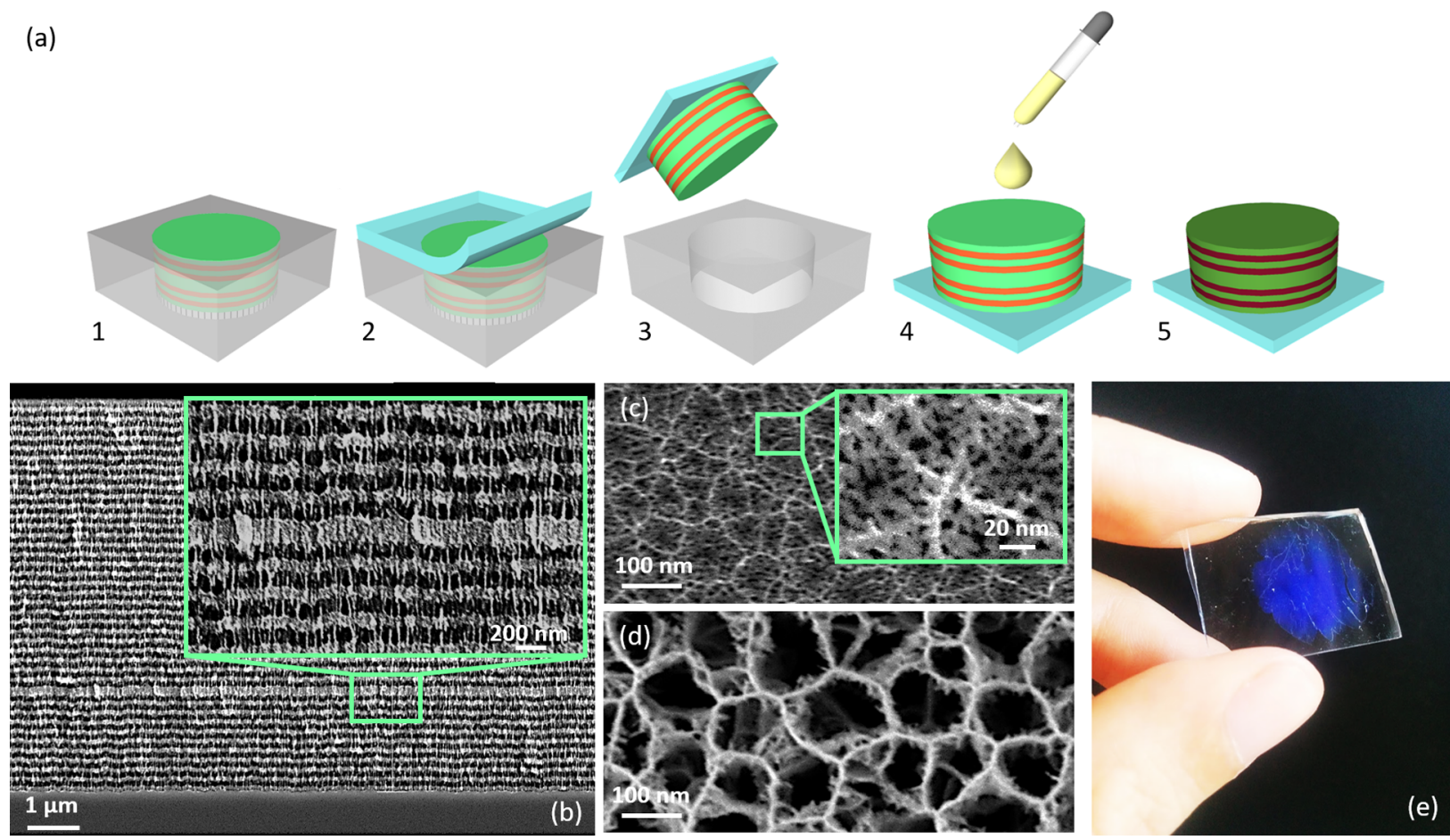

Figure 1. Fabrication of the hybrid PSi/PFO laser. (a) Preparation scheme of PFO-infiltrated PSi resonant microcavity: (1) etching of an asymmetric PSiMC and subsequent thermal oxidation; (2) application of a thin PDMS slab on top of the silicon chip; (3) peeling of the PSiMC off the silicon chip through the PDMS slab; (4) infiltration of the PSiMC with PFO via drop-casting; (5) hybrid PSi/PFO resonant cavity resulting after solvent evaporation. (b) SEM cross-section image of an as-prepared PSiMC with 30 and 10 bilayers on top and at the bottom, respectively, of the defect layer. The alternating darker/brighter regions correspond to high/low-porosity (low/high refractive index) layers. Inset: Magnification of the region around the defect layer. (c) SEM top-view image detailing the morphology of low-porosity $(P=59 \%)$ layers of the PSiMC. Inset: Magnification highlighting pore size (about $10 \mathrm{~nm}$ ) and distribution. (d) SEM top-view image detailing the morphology of high-porosity $(P=77 \%)$ layers of the PSiMC, with a pore size of about $100 \mathrm{~nm}$. (e) Photo of an asymmetric PSiMC with resonance wavelength at $460 \mathrm{~nm}$, transfer-printed onto a PDMS slab.

finely controlling light propagation, and resonant microcavities, ${ }^{22,23}$ either monolithic or transfer-printed, able to achieve strong light emission modification of emitter compounds have been reported. However, the presence of silicon, which has a strong absorption in the visible spectrum, has restricted the applicability of these PSi optical structures to the (near) infrared region.

In this scenario, there is nowadays an increasing effort put into the preparation of nonabsorbing PSi optical structures suitable for the UV-vis applications. Recently, Braun et al. reported on the fabrication of transparent porous silicon dioxide $\left(\mathrm{PSiO}_{2}\right)$ and $\mathrm{PSiO}_{2} /$ titanium dioxide $\left(\mathrm{TiO}_{2}\right)$ optical elements, by either thermal oxidation or using a combination of thermal oxidation and atomic layer deposition infilling of $\mathrm{PSi}^{24,25}$

In spite of such an intense effort, to the best of our knowledge, the original dream of a PSi-based laser with roomtemperature operation, either in the visible or in the nearinfrared region, has not been demonstrated yet.

In this work, we report on room-temperature lasing from monolithically integrated PSi microcavities. The laser structure consists of a high-quality, asymmetric and fully transparent PSiMC that is able to support blue lasing action from a conjugated polymer, e.g., poly $(9,9)$-dioctylfluorene (PFO), infiltrated inside it. The PFO-infiltrated PSiMC features single-mode blue lasing at the resonance wavelength of 466 $\mathrm{nm}$, with a line width of $\sim 1.3 \mathrm{~nm}$ and lasing threshold as low as $5 \mathrm{~nJ}$ (i.e., fluence of $15 \mu \mathrm{J} / \mathrm{cm}^{2}$ ), which is among the lowest values of state-of-the-art PFO-based lasers. ${ }^{26-28}$ Time-resolved photoluminescence further highlights a substantial modification of the emission lifetime in the PSiMC, which is shortened by $57 \%$ within the cavity mode, thus confirming a dramatic variation of the radiative decay rate due to a Purcell effect.

\section{RESULTS AND DISCUSSION}

Fabrication of Hybrid PSi/PFO Laser. The preparation method of the hybrid PSi/PFO laser is sketched in Figure 1(a). First, we prepared a monolithic asymmetric PSi microcavity operating in the visible region and consisting of a halfwavelength defect sandwiched between two quarter-wavelength DBRs featuring a different number of bilayers (Figure 1a-1). The PSiMC is fabricated by a single-step electrochemical etching, in a $\mathrm{HF} / \mathrm{H}_{2} \mathrm{O}$ electrolyte, of a highly doped p-type crystalline silicon. A square-wave etching current density profile with values of 20 and $400 \mathrm{~mA} \mathrm{~cm}{ }^{-2}$ is used to produce a stack of alternating PSi layers with porosity values of $59 \%$ (i.e., 1.55 refractive index unit (RIU) at $550 \mathrm{~nm}$ ) and 77\% (1.28 RIU), respectively. These values represent a trade-off among achieving a porosity variation that maximizes the refractive index contrast, avoiding the collapse of the PSi at higher porosity due to mechanical instability, and eluding reduced penetration of polymer in the PSiMC due to the small diameter of the pores at lower porosity. Interestingly, a linear relationship is found between the central wavelength of the DBR stop-band, and, in turn, the resonance wavelength of the cavity, and the temporal period of the etching current density square-wave (Supporting 

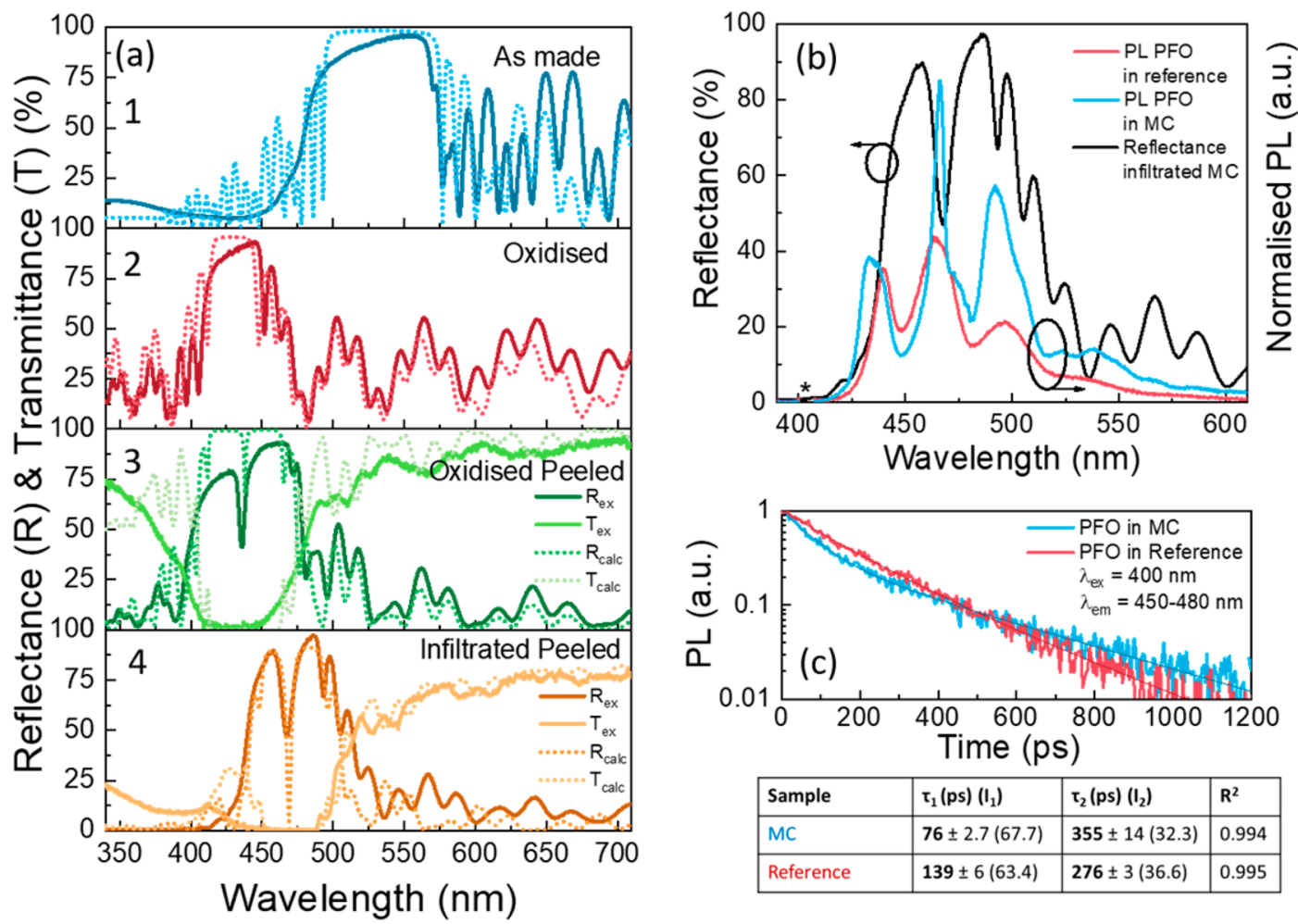

Figure 2. Spectroscopic characterization of the PSi/PFO microcavity. (a) Experimental (solid lines) and calculated (dotted lines) reflectance (R) and transmittance (T) spectra of PSiMC at each preparation step: (1) reflectance spectrum of the as-prepared microcavity; (2) reflectance spectra of the microcavity after the thermal oxidation; (3) reflectance (dark green line) and transmittance (light green line) spectra of the microcavity transfer-printed onto the PDMS slab; (4) reflectance (dark orange line) and transmittance (light orange line) spectra of the microcavity after infiltration of PFO. (b) Peak-normalized PL spectra under continuous wave (CW) laser excitation $(\lambda=405 \mathrm{~nm}$, indicated with an "*” in (b)) of PFO when infiltrated into the transfer-printed PSiMC (blue line) and into a PSi layer with a constant porosity of $66 \%$ used as reference nonresonant structure (red line). The reflectance spectrum (black line) of the PFO-infiltrated PSiMC is also reported superposed to the emission spectra. (c) Typical PL radiative decays of PFO infiltrated in the microcavity (blue line) and in the PSi nonresonant structure used as reference (red line) at wavelengths corresponding to the cavity mode $\left(\lambda_{\mathrm{ex}}=400 \mathrm{~nm}, \lambda_{\mathrm{em}}=450-480 \mathrm{~nm}\right)$; PL decay time constants are reported in the table.

Information Section 1). In fact, by increasing (decreasing) the temporal period of the etching current square-wave it is possible to increase (decrease) the optical thickness of high and low porosity layers in the PSiMC, which leads to a red(blue)shift of the DBR stop-band and enables, in turn, the fine-tuning of the resonance wavelength of the cavity over the whole visible range (Supporting Information Section 1).

Figure $1 \mathrm{~b}$ shows scanning electron microscopy (SEM) crosssection images highlighting the architecture of an as-prepared asymmetric PSiMC with a half-wavelength defect layer sandwiched between two DBRs with 30 (top) and 10 (bottom) bilayers. The square-wave modulation of the porosity along the direction perpendicular to the wafer surface can be clearly appreciated, with darker/brighter alternating layers indicating high/low porosity (low/high refractive index) regions. The defect layer of the cavity can be better appreciated in the inset, also highlighting the columnar structure of the pores running perpendicularly to the wafer surface. The detailed morphology of both low- and high-porosity layers of the PSiMC is shown in the top-view SEM images in Figure $1(\mathrm{c}, \mathrm{d})$, from which pore sizes in the range of $10-20$ and $100-120 \mathrm{~nm}$, respectively, are apparent.

After the PSiMC is fully etched, the etching current density value is abruptly increased to produce pseudo-electropolishing (porosity about 98\%) of the silicon underneath the cavity, which facilitates its eventual transfer-printing to a different substrate, though ensuring that enough silicon is left to mechanically hold the cavity in place during the next hightemperature thermal oxidation. The latter is necessary to fully convert silicon into silicon dioxide, and eliminate, in turn, any silicon-induced absorption in the UV-vis region. Pictures of asymmetric PSiMCs both as-prepared and oxidized are given in Supporting Information Section 2.

We then transfer-printed the oxidized PSiMC onto a transparent material by directly peeling the cavity off the silicon substrate using an elastomeric film, namely, polydimethylsiloxane (PDMS), to enable transmittance operation (Figure 1a-2). The high chemical affinity between silicon dioxide and PDMS ensures an easy and effective transfer of the PSiMC from the silicon chip to the PDMS film, with a damagefree and up-scalable approach (Figure 1a-3). Figure 1e shows a picture of an asymmetric PSiMC, with resonance wavelength at $460 \mathrm{~nm}$, transfer-printed onto a PDMS slab. The uniformity of the blue color reflected from the cavity is an indication of the good reliability of the whole preparation process, in terms of etching, oxidation, and transfer-printing steps.

Eventually, infiltration of a blue-emitting polymer, namely, $\mathrm{PFO}$, inside the cavity is carried out via drop-casting of the polymer solution into the transfer-printed PSiMC (Figure 1a4). The polymer solution uniformly infiltrates the PSi structure for its full depth, resulting in a hybrid (i.e. inorganic-organic) PSi/PFO resonant cavity after solvent evaporation (Figure 1a- 
5). Fluorescence microscope images of the PSiMC after PFO infiltration highlight a good uniformity of the infiltration process, both in the in-plane and in the out-of-plane directions of the cavity (Supporting Information Section 2).

Spectroscopic Characterization of PSi/PFO Microcavity. Typical reflectance and transmittance experimental spectra collected at each preparation step of the PSi/PFO hybrid microcavity are shown in Figure 2a. Figure 2a-1 shows the reflectance spectrum at normal incidence of the as-prepared PSiMC (step 1). The photonic stop-band is centered at 520 $\mathrm{nm}$, with a full-width at half-maximum (fwhm) of $85 \mathrm{~nm}$ and reflectance above $90 \%$. Reflectance fringes above $575 \mathrm{~nm}$ are due to Fabry-Perot interference in the PSi layer. Notice that the reflectance notch corresponding to the resonance wavelength of the cavity (defect mode), which should occur at 525 $\mathrm{nm}$ by design, is not visible in the reflectance spectrum (blue solid line). This is due to the uneven number of bilayers of DBRs at each side of the cavity. In fact, the asymmetric architecture consists of an actual resonant cavity with two DBRs with 10 bilayers on each side of the defect layer plus an additional DBR with 20 bilayers on top of the MC. The latter dominates the reflectance spectrum of the on-chip asymmetric PSiMC, hindering the actual resonant cavity underneath, as confirmed by the theoretical reflectance spectrum calculated using the transfer matrix method (TMM) (light blue dotted line).

After the oxidation process, the photonic stop-band blueshifts about $90 \mathrm{~nm}$ due to a lowering of the refractive indices of both high- and low-porosity layers (Figure 2a-2, red line). ${ }^{29}$ The fwhm of the photonic stop-band reduces to $45 \mathrm{~nm}$, in agreement with the lowered refractive index contrast, though a high reflectance $(>90 \%)$ is maintained. The theoretical spectrum (dark red dotted line) confirms that more than the $99 \%$ of the silicon is converted into silicon dioxide. This is also confirmed by Fabry-Perot interference fringes visible at wavelengths below $450 \mathrm{~nm}$ in the oxidized PSiMC, where silicon absorption dominated for as-prepared PSiMCs.

Both reflectance and transmittance spectra of the oxidized PSiMC after transfer-printing on a PDMS slab are reported in Figure 2a-3 (green and light green solid lines). In this case, the reflectance spectrum is collected on the opposite side of the PSiMC compared to the previous cases, with the reflectance probe facing the DBR with 10 bilayers. As expected, the reflectance spectrum (dark green line) now shows a sharp notch at $420 \mathrm{~nm}($ fwhm $=4.1 \mathrm{~nm})$ with a quality factor $Q=\lambda$ / fwhm of 102.5, and the transmittance spectrum (light green line) of the PSiMC shows a dip in correspondence of the photonic stop-band. The cavity mode does not show up in the transmittance spectrum, as validated by theoretical calculations (green and dark green dotted lines). We argue that light traveling through the cavity at the resonance wavelength is reflected back by the DBR with 20 bilayers, though scattered traveling back and forth in the cavity. The cavity shows a transmittance higher than $75 \%$ over the entire visible range outside the photonic band gap, which further confirms PSi full oxidation.

The infiltration of PFO in the transfer-printed PSiMC results in a red-shift (about $50 \mathrm{~nm}$ ) of the photonic stop-band, due to the increase in the effective refractive index of the structure induced by the presence of a polymer with a refractive index greater than that of air, ${ }^{30}$ with a concomitant increase of the fwhm (about $7 \mathrm{~nm}$ ) of the defect mode at $466 \mathrm{~nm}(Q=67)$, due to the lowered refractive index contrast (Figure 2a-4, orange and light orange solid lines). The stop-band remains well-defined with no additional shoulders, indirect indication that the cavity is uniformly infiltrated with PFO. The transmittance value is still higher than $55 \%$ at wavelengths above $500 \mathrm{~nm}$, though a broad absorption band emerges below $400 \mathrm{~nm}$ that further confirms the presence of the PFO. Theoretical spectra (orange and light orange dotted lines) point out that the fraction of the polymer infiltrated in the PSiMC is $4 \%$ of the air volume.

The good agreement between experimental and calculated spectra after each preparation step confirms good accuracy between design and fabrication of PSi/PFO microcavities, for both morphological (i.e., layer thickness, porosity, period) and optical (i.e., refractive index value, profile) parameters. A more detailed description of the calculated spectra is reported in the Supporting Information Section 2.

Figure $2 \mathrm{~b}$ compares peak-normalized emission spectra under continuous wave (CW) laser excitation $(\lambda=405 \mathrm{~nm})$ of PFO when infiltrated into the transfer-printed PSiMC (blue line) and into an oxidized PSi layer with constant porosity of $66 \%$ transfer-printed on a PDMS slab (red line, used as reference nonresonant structure). The reflectance spectrum (black line) of the PFO-infiltrated PSiMC is also reported in Figure $2 \mathrm{~b}$ superposed to the emission spectra. The PSi reference acts as a simple interferometer (reflectance spectrum in Supporting Information Section 3), and it does not provide any optical feedback that might induce modification of the density of state of PFO. The PL spectrum of PFO infiltrated inside the PSi reference exhibits the characteristic vibronic progression of the emission peaks at 438,466 , and $497 \mathrm{~nm}$. This is fully consistent with the photoluminescence spectrum of a PFO thin film dropcast onto a PDMS slab (Supporting Information Section 3) and in perfect agreement with the one reported in the literature for the $\beta$-phase of the polymer. ${ }^{31}$

On the other hand, when PFO is embedded inside the PSiMC, which was designed to have the cavity mode perfectly overlapping the amplified spontaneous emission (ASE) of $\mathrm{PFO},{ }^{26}$ there is a redistribution of the oscillator strength ${ }^{17}$ that strongly modifies the PL spectrum. Specifically, the PL emission is significantly enhanced (50\%) and narrowed (fwhm $=2.9 \mathrm{~nm}$ ) in correspondence with the cavity mode wavelength $\left(\lambda_{\mathrm{em}}=466 \mathrm{~nm}\right.$, in the reflectance spectrum) due to a rearrangement of the photonic density of states (DOS) (Supporting Information Section 4). ${ }^{32}$ This is confirmed by time-resolved PL (TRPL) measurements carried out using a pulsed laser source $\left(\lambda_{\text {excitation }}=400 \mathrm{~nm}\right)$ with low excitation power $(10-50 \mathrm{nW})$ at room temperature. Figure $2 \mathrm{c}$ shows typical PL radiative decays of PFO infiltrated in the cavity at wavelengths corresponding to the cavity mode $(\lambda=450-480$ $\mathrm{nm})$; PL decay time constants and intensities are reported in the table. TRPL shows a faster decay time of PFO emission when infiltrated inside the PSiMC (blue line) than when infiltrated inside the PSi reference (red line). It is worth restating that the reference PSi structure consists of a simple porous silicon layer with constant porosity (i.e., constant refractive index) that does not provide any photonic effect on the emitting material and its DOS.

Interestingly, the PL temporal decay curve is best fit by a biexponential function $\left(I(t)=I_{0}+I_{1} \mathrm{e}^{-t / \tau 1}+I_{2} \mathrm{e}^{-t / \tau 2}\right)$. The need for a biexponential fitting is not surprising for the infiltrated polymer, given the large surface/volume ratio of the regions in which the polymer is embedded, which can strongly influence the physical properties of the emitter. ${ }^{33}$ This is in agreement 

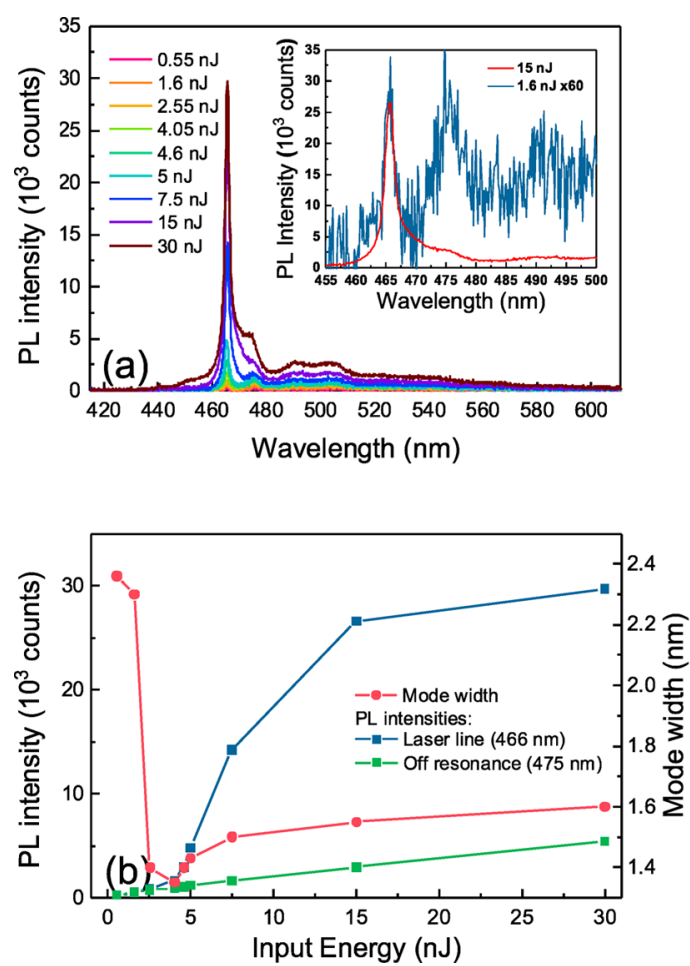
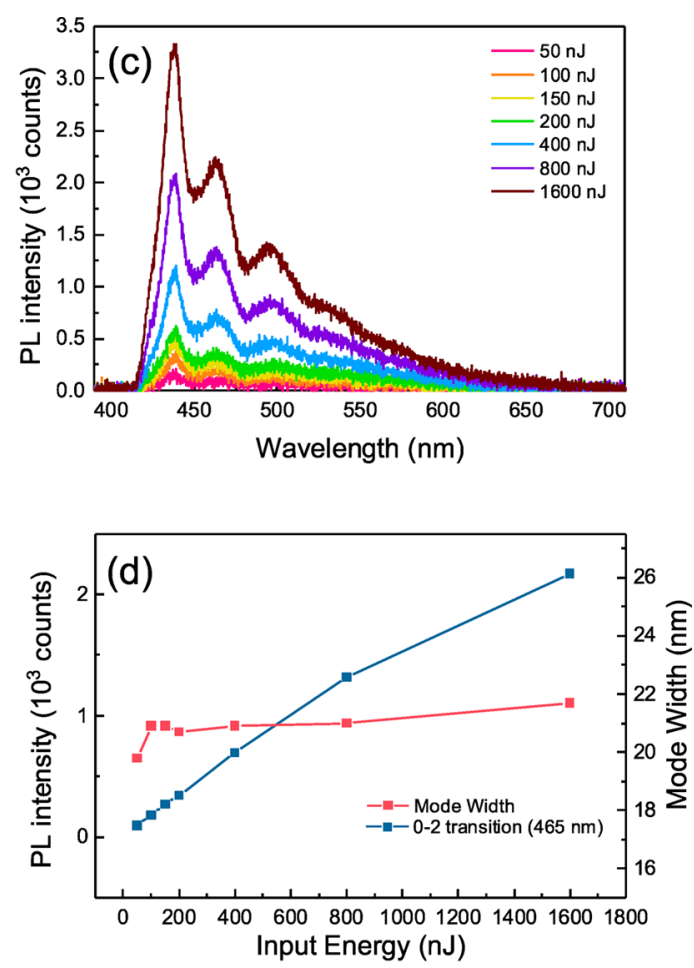

Figure 3. Lasing action from PSi/PFO microcavities. (a) PL spectra of an asymmetric PFO-infiltrated PSiMC for different pump energies per pulse in the range 0.55 to $30 \mathrm{~nJ}$. Inset: PL spectra collected below (blue line, intensity values are multiplied $\times 60)$ and above (red line) the lasing threshold. (b) PL intensity (blue squares) and mode width (fwhm) (red dots) of the laser line (at $466 \mathrm{~nm}$ ) of the PFO-infiltrated PSiMC as a function of the input energy. Above an excitation energy per pulse of $5 \mathrm{~nJ}\left(\right.$ i.e., fluence of $\left.15 \mu \mathrm{J} / \mathrm{cm}^{2}\right)$ the peak at $466 \mathrm{~nm} \mathrm{narrows}$ $(\mathrm{fwhm}=1.35 \mathrm{~nm}$ at $5 \mathrm{~nJ})$ and its intensity increases superlinearly with the pump energy. The intensity of the emission measured outside the cavity mode (at $475 \mathrm{~nm}$ ) is significantly lower than that at $466 \mathrm{~nm}$ and increases linearly with the pump energy. (c) PL spectra of PFO when infiltrated in the PSi reference with constant porosity, for different pump energies per pulse in the range 50 to $1600 \mathrm{~nJ}$, showing a line shape similar to that measured under CW excitation regardless of the pump energy value. (d) PL intensity (blue squares) and mode width (red squares) of PFO when infiltrated in the PSi reference, measured at $465 \mathrm{~nm}$, corresponding to the 0-2 transition. PL intensity increases linearly with the pump energy, and no line narrowing occurs for the PSi reference.

with the physical model of the luminescence of conjugated polymers originating from both strongly bound intramolecular excitons (primary exponential) and more weakly bound interchain states (secondary exponential, with a longer decay time).$^{34-39}$ In our system the longer decay time can be assigned either to the polymer interaction with the $\mathrm{SiO}_{2}$ nanostructure or to the formation of polymer aggregates inside the pores. The time-constant values of PL reported in Figure 2 provide clear evidence of the modification of the decay rates induced by the photonic feedback structure. Specifically, we observe a significant reduction $\left(\sim 57 \%\right.$ for $\left.\tau_{1}\right)$ of the primary exponential decay time, which can be ascribed to a modified exciton recombination time when the polymer is infiltrated in the PSiMC and confirms the rearrangement of the DOS inside the cavity at the resonance wavelength. Calculation of the ratio between spontaneous emission lifetime of a dipole embedded in a nonresonant medium and of a dipole located inside the microcavity at the lasing/resonance wavelength yields $\tau_{1 \text { Ref }} /$ $\tau_{1 \mathrm{MC}} \approx 1.83$ (obtained from experimental data in Figure $2 \mathrm{c}$, table), from which a Purcell factor $F_{\mathrm{p}} \approx 1.9$ is estimated according to the literature. ${ }^{40-42} \mathrm{~A}$ value of $F_{\mathrm{p}}>1$ supports the enhancing of spontaneous emission rate in resonant microcavities $^{32,43}$ through a Purcell effect. ${ }^{44,45}$

The redistribution of the DOS induced by the PSiMC is further corroborated by the observation of slower decay time of the PL at wavelengths outside the cavity mode in correspondence with the photonic stop-band, compared to that on the PSi reference (Supporting Information Section 4).

Modification of the density of states of the PFO host in PSiMCs and the relatively high $Q$ of PSiMCs is a clear indication that porous silicon microcavities represent a suitable feedback structure for an organic laser.

Lasing Action from PSi/PFO Microcavity. Lasing of PFO-infiltrated PSiMC is investigated using a Ti-sapphire laser $\left(\lambda_{\mathrm{ex}}=400 \mathrm{~nm}, 150 \mathrm{fs}, 2 \mathrm{kHz}\right.$, spot diameter $\left.100 \mu \mathrm{m}\right)$ as a pumping source and collecting the emission spectra as a function of pump power at normal incidence. A sketch of the experimental setup used is shown in Supporting Information Section 5. Specifically, the PFO-infiltrated PSiMC was placed with the 30-bilayer DBR facing the laser pump and the 10bilayer DBR facing the collection lens. This configuration permits maximizing the emission of PFO inside the cavity in the collection direction. In fact, light emitted from PFO inside the cavity at the resonant wavelength in the direction of the laser pump is reflected back to the collection direction from the uneven 20-bilayer of the DBR.

Figure 3a shows the experimental PL spectra of an asymmetric PFO-infiltrated PSiMC for pump energies per pulse from 0.55 to $30 \mathrm{~nJ}$. At low excitation energies (below 2 nJ, inset in Figure 3a) the PL spectrum shows a sharp peak at $466 \mathrm{~nm}$ with $\mathrm{fwhm}=2.4 \mathrm{~nm}$, which corresponds to the defect mode wavelength of the PFO-infiltrated PSiMC, in good agreement with the PL spectrum under CW excitation of 
Figure 2(e). A broader PL band is observed at $475 \mathrm{~nm}$, which corresponds to the unmodified emission of PFO, with intensity comparable to that of the cavity mode at $466 \mathrm{~nm}$. Above a pump energy of $5 \mathrm{~nJ}$ (corresponding to a fluence of $\sim 15 \mu \mathrm{J} /$ $\mathrm{cm}^{2}$ ) the peak at $466 \mathrm{~nm}$ narrows (fwhm $=1.35 \mathrm{~nm}$ at $5 \mathrm{~nJ}$ ) and its intensity increases nonlinearly with the excitation energy. This is apparent in Figure 3(b), which shows both output power and mode width (fwhm) of the laser line (466 $\mathrm{nm}$ ) of the PFO-infiltrated PSiMC as a function of the input pump energy. The PL emission at $466 \mathrm{~nm}$ is best fit with two Gaussian curves, both centered at $466 \mathrm{~nm}$, with the higher intensity, sharper component assigned to the single mode laser emission of PFO infiltrated into the PSiMC and the lower intensity, broader component (fwhm $\sim 6-8 \mathrm{~nm}$ ) assigned to unmodified PL collected by the collimation lens in our experimental setup.

We claim that the strong nonlinear increase of the PL intensity at $466 \mathrm{~nm}$, together with the perfect matching of the sharp PL emission line and cavity resonant mode (both at 466 $\mathrm{nm}$ ), is a clear indication of lasing action from PFO infiltrated inside the PSiMC. Accordingly, we identify the excitation energy per pulse of $5 \mathrm{~nJ}\left(\sim 15 \mu \mathrm{J} / \mathrm{cm}^{2}\right)$ as the lasing threshold of our PFO-infiltrated PSiMC. This is in good agreement with the theoretical value calculated in Supporting Information Section 6. Interestingly, this value is comparable to that of best state-of-the-art PFO lasers, ${ }^{26-28}$ thus confirming the high quality of the photonic feedback structure achieved using porous silicon.

Note that the PL intensity at $475 \mathrm{~nm}$ (green line and square, Figure 3(b)) always increases linearly with the pump energy, thus confirming that no stimulated emission (i.e., only spontaneous emission) occurs outside the resonant mode of the cavity. The ratio between PL intensity of resonant and nonresonant modes ${ }^{43,46}$ (i.e., between stimulated and spontaneous emission, Supporting Information Section 6) further confirms the lasing threshold of about $5 \mathrm{~nJ}\left(15 \mu \mathrm{J} / \mathrm{cm}^{2}\right)$.

At high input energies, we observe that the width of the laser line slightly increases upon increasing the excitation fluence (Figure 3(b)), which is a fingerprint for pulsed pumped organic lasers operating in the gain switching regime. ${ }^{47,48}$

To evaluate the operating stability of the hybrid PFO/PSi laser with time, the PFO-infiltrated PSiMC was irradiated with a laser energy of $50 \mathrm{~nJ}$, which is 10 times higher than the lasing threshold, yielding only a $\sim 40 \%$ decay of the PL intensity at the lasing wavelength after $6 \mathrm{~min}$ of irradiation (i.e., $7.2 \times 10^{5}$ pulses). These values are in good agreement with organic semiconductor and solid-state dye lasers, whose operating lifetimes are typically on the order of $10^{5}$ to $10^{7}$ pulses ${ }^{49}$ (Supporting Information Section 7).

To rule out that the observed effect on the PFO-infiltrated PSiMC is related to ASE, PL spectra are also collected at the output of the PFO-infiltrated PSi reference for excitation energy per pulse from 50 to $1600 \mathrm{~nJ}$ using the same experimental setup. Figure $3 \mathrm{c}$ shows that the PL spectra of PFO when infiltrated in the PSi reference with constant porosity have a line shape similar to that measured under CW excitation (shown in Figure $2 \mathrm{~b}$ ), regardless of the excitation energy (Supporting Information Section 8). Furthermore, PL intensity increases linearly with the pump energy, and no line narrowing occurs for the PSi reference (Figure 3d).

Furthermore, lasing from PFO-infiltrated PSiMC is also observed for a microcavity designed to have a resonance wavelength red-shifted $(591 \mathrm{~nm})$ with respect to PFO emission
(Supporting Information Section 9). In this case, the PSiMC was properly rotated by $50^{\circ}$ to blue-shift the stop-band thanks to angular dispersion of photonic crystal structures so as to overlap the emission spectrum of the PFO (Supporting Information Section 9). This result further corroborates high quality in fabrication and good flexibility in operation of the proposed PSi-based laser.

\section{CONCLUSION}

In this work we reported on a ground-breaking approach for the fabrication of a monolithic low-threshold silicon-based hybrid laser via drop-casting infiltration of transparent nanostructured porous silicon resonant cavities with a polymer emitter.

As a proof-of-concept, fabrication of a blue-emitting hybrid laser by infiltration of PFO within a PSiMC is successfully reported. The laser features a single mode laser line at $466 \mathrm{~nm}$ (mode width $\sim 1.3 \mathrm{~nm}$ ) with an exceptionally low threshold (input energy per pulse $\sim 5 \mathrm{~nJ}$, fluence $\sim 15 \mu \mathrm{J} / \mathrm{cm}^{2}$ ). Furthermore, a significant modification of the density of states of PFO when infiltrated in the PSiMC indicates that a Purcell effect also takes place.

The laser fabrication is low-cost, highly reliable, and upscalable. It is based on the preparation of a monolithic PSiMC by one-step electrochemical etching, subsequent thermal oxidation, transfer-printing to a PDMS slab, and final dropcasting of PFO into the microcavity. The laser fabrication is also very flexible. In fact, it is possible to effectively tune the laser wavelength by simply shifting the PSiMC resonant wavelength and infiltrating a suitable polymer with emission matching the resonant mode of the PSiMC. Moreover, patterning of the microcavity down to a few micrometers in size can be carried out by standard photolithography.

These results are significant for the development of low-cost, low-threshold silicon-based lasers operating from the visible to the near-infrared range, which could be exploited in many applications, including silicon photonics, ultrasensitive biosensors, and personalized nanomedicine. Electrical pumping, for which the majority of the aforementioned applications would benefit from, remains a challenging goal to be achieved with hybrid lasers though, due to both low carrier mobility and triplet generation in organic optical-gain materials. Nevertheless, the proposed PSi/organic hybrid laser could represent an effective proof-of-concept platform for the development of indirect electrically-pumped lasers, where an efficient electrically-driven light source is used to optically pump the PSi/ organic laser, leveraging the high quality of the PSi feedback structure and, in turn, the low-energy lasing threshold exhibited by this class of lasers.

\section{METHODS}

Preparation of PSiMC and PSi Reference. Porous silicon microcavities and PSi reference interferometers were prepared by anodic etching of highly doped p-type crystalline silicon (borondoped, $\langle 100\rangle$-oriented, resistivity of $0.8-1.2 \mathrm{~m} \Omega \mathrm{cm}$, Siltronix, Inc.). The electrochemical etching was carried out in a homemade Teflon cell using a two-electrode configuration, with the silicon substrate (area of $2.835 \mathrm{~cm}^{2}$ ) placed onto an aluminum flat anode and a platinum coil immersed in the solution of HF $(48 \%) / \mathrm{EtOH}(3: 1 \mathrm{v} / \mathrm{v})$ and acting as cathode. A source measure unit (SMU) (Keithley 2610), configured as current source and voltage meter, was used to set the etching current density, both value and profile, and simultaneously monitor the voltage drop between silicon and platinum electrodes. 
First, a PSi sacrificial layer was etched at a constant current density of $400 \mathrm{~mA} / \mathrm{cm}^{2}$ for $15 \mathrm{~s}$ and immediately dissolved by dissolution in a $\mathrm{NaOH}(1 \mathrm{M}$ in deionized (DI) water)/EtOH $(9: 1 \mathrm{v} / \mathrm{v})$ solution. This step is necessary to avoid the presence of a top parasitic porous silicon layer, with pores smaller than $10 \mathrm{~nm}$ due to chemical contamination of the silicon surface, which could strongly impact the preparation of PSi optical structures. After removal of the PSi sacrificial layer, the silicon samples were rinsed with deionized water and ethanol, gently dried under nitrogen flow, then used for the etching of a second PSi optical structure, either the PSiMC or the PSi reference interferometer.

For the etching of the PSiMC we set a square-wave current density profile with values of 400 and $20 \mathrm{~mA} / \mathrm{cm}^{2}$ to obtain a periodic modulation of the PSi porosity between $77 \%$ and $59 \%$, respectively, and, in turn, of the PSi refractive index varying between $n_{400}=1.28$ RIU and $n_{20}=1.55 \mathrm{RIU}$ (at $550 \mathrm{~nm}$ ), yielding a contrast of $0.27 \mathrm{RIU}$. The porosity values and, in turn, the refractive index values of the PSi layers prepared with the aforementioned current densities were extracted from reflectance spectra collected from PSi homogeneous layers etched at constant current density and, in turn, with constant porosity. ${ }^{50}$

The PSiMC was designed with a half-wavelength defect sandwiched between quarter-wavelength DBRs with 30 (top) and 10 (bottom) bilayers. The PSiMC with stop-band at $525 \mathrm{~nm}$ as-prepared was etched using a square-wave etching current profile with a period of $4.5 \mathrm{~s}$ and duty cycle of 0.9 (i.e., $t_{400}=4.95 \mathrm{~s}, t_{20}=0.55 \mathrm{~s}$ ), to take into account the different etching speeds at different etching current densities. The etching time of the two current density values is tuned to ensure that the optical thickness of both high- and low-porosity layers is onequarter of the central wavelength of the photonic stop-band of the DBRs. Notice that, the etching time is doubled during the etching of the defect layer of the cavity to obtain an optical thickness that is half the stop-band central wavelength. The defect layer is etched at $20 \mathrm{~mA} /$ $\mathrm{cm}^{2}$ to have a low porosity value and, in turn, a high refractive index value that allows the electromagnetic field to be concentrated inside the cavity. The overall etching process gives rise to a PSiMC with a total thickness of $\sim 15 \mu \mathrm{m}$.

The PSi reference interferometer was prepared at a constant etching current of $100 \mathrm{~mA} / \mathrm{cm}^{2}$ for $350 \mathrm{~s}$ to obtain a homogeneous PSi layer with constant porosity of $66 \%$ and thickness of $\sim 15 \mu \mathrm{m}$, comparable to average porosity and total thickness of the PSiMC.

After preparation of the PSi optical structures, we applied a constant current density of $800 \mathrm{~mA} / \mathrm{cm}^{2}$ for $0.6 \mathrm{~s}$ to produce a pseudoelectropolishing of the silicon underneath the cavity, though leaving the microcavities still anchored to bulk silicon. The as-prepared samples are then rinsed with 2-propanol and pentane and gently dried under nitrogen flow to obtain a crack-free PSi layer.

Eventually, samples are thermally oxidized in a muffle furnace (ZB 1, ASAL) at $750{ }^{\circ} \mathrm{C}$ for $1: 30 \mathrm{~h}$, with a ramp rate of $12{ }^{\circ} \mathrm{C} / \mathrm{min}$, in ambient conditions.

Transfer-Printing of PSiMC onto PDMS. PDMS slabs (Sylgard 184, Dow Corning, Midland, MI, USA) were prepared by mixing base and curing agent in a ratio 10:1 by weight. The resulting mix was cast onto a Petri dish and degassed under vacuum for $30 \mathrm{~min}$ to avoid the presence of air bubbles trapped inside it. The Petri dish containing the mix was then placed into an oven at $110^{\circ} \mathrm{C}$ for $1 \mathrm{~h}$ to achieve polymer cross-linking. Eventually, the PDMS disks were properly cut into slabs, which were placed onto the silicon chips containing either the oxidized PSiMC or the PSi reference and used to gently peel the PSi structures off the silicon substrate and transfer them onto the PDMS slab.

Infiltration of PFO into PSiMC. Poly(9,9)-dioctylfluorene (20 $\mathrm{kDa}$. Aldrich) was used as received, without further purification. A PFO solution was prepared by dissolving $1 \%$ wt in toluene at room temperature. The infiltration was performed by dropping $20 \mu \mathrm{L}$ of the polymer solution onto the PSiMC, then allowing the solvent to evaporate in ambient conditions.

Morphological and Optical Characterization of PSi/PFO Microcavities. Reflectance and transmittance spectra were collected with an Ocean Optics H4000+ES spectrometer in combination with a fiber-coupled Ocean Optics DH-2000 deuterium-halogen source. CW photoluminescence measurements were carried out with the same spectrometer by using a $405 \mathrm{~nm}$ CW laser diode (Thorlabs). The porosity of the PSi layers was computationally evaluated by fitting experimental reflectance spectra of as-prepared PSi samples with a homemade software developed in Matlab (MathWorks, USA). Timeresolved PL measurements were carried out using a femtosecond laser source coupled to a streak camera detection system (Hamamatsu C5680). A Ti:sapphire laser (Coherent Chameleon Ultra II, pulse bandwidths of $\sim 100 \mathrm{fs}$, repetition rate of $80 \mathrm{MHz}$, and power of $10-$ $50 \mathrm{nW}$ ) was used to pump a second-harmonic crystal (barium borate) to tune the pump wavelength to $400 \mathrm{~nm}$.

Lasing experiments were performed using an amplified Ti:sapphire laser with $2 \mathrm{~mJ}$ output energy and $2 \mathrm{kHz}$ repetition rate at $400 \mathrm{~nm}$. The laser power was set using a tunable optical density. The samples were placed with the 30-bilayer stack facing the focused laser pump and the 10-bilayer stack facing the collection lens. The emitted light was collected through a spherical collimation lens connected to a fibercoupled spectrometer (Avantes AvaSpec-ULS2048XL-EVO).

SEM images were taken using a Zeiss ULTRA55 microscope.

\section{ASSOCIATED CONTENT}

\section{Supporting Information}

The Supporting Information is available free of charge on the ACS Publications website at DOI: 10.1021/acsnano.8b00875.

Additional experiments, figures, and data on morphological characterization of PSi layers and PSiMCs; spectroscopic investigation of PSiMCs and PSi reference structure before and after infiltration of PFO; spectroscopic characterization of PFO solution and thin film; Fermi's golden rule and radiative decay rate; lasing experiment setup; theoretical estimation of lasing threshold and coherence length; operating stability of $\mathrm{PFO} / \mathrm{PSiMC}$ laser; spectroscopic characterization of the red-shifted PSiMC (PDF)

\section{AUTHOR INFORMATION}

\section{Corresponding Author}

*E-mail: g.barillaro@iet.unipi.it.

ORCID

Giuseppe M. Paternò: 0000-0003-2349-566X

Giuseppe Barillaro: 0000-0001-6197-4851

Notes

The authors declare no competing financial interest.

\section{ACKNOWLEDGMENTS}

This work was supported by the EU H2020 ETN SYNCHRONICS under grant agreement 643238. We thank Dr. F. F. R. Toia (STMicroelectronics, Milan) for technical assistance with SEM images.

\section{REFERENCES}

(1) Cullis, A. G.; Canham, L. T. Visible Light Emission Due to Quantum Size Effects in Highly Porous Crystalline Silicon. Nature 1991, 353, 335.

(2) Canham, L. Gaining Light From Silicon. Nature 2000, 408, 411412 .

(3) Liang, D.; Bowers, J. E. Recent Progress in Lasers on Silicon. Nat. Photonics 2010, 4, 511.

(4) Priolo, F.; Gregorkiewicz, T.; Galli, M.; Krauss, T. F. Silicon Nanostructures for Photonics and Photovoltaics. Nat. Nanotechnol. 2014, 9, 19.

(5) Lin, V. S.; Motesharei, K.; Dancil, K. P.; Sailor, M. J.; Ghadiri, M. R. A Porous Silicon-Based Optical Interferometric Biosensor. Science 1997, 278, 840-843. 
(6) Mariani, S.; Pino, L.; Strambini, L. M.; Tedeschi, L.; Barillaro, G. 10 000-Fold Improvement in Protein Detection Using Nanostructured Porous Silicon Interferometric Aptasensors. ACS Sens 2016, 1, 14711479 .

(7) Gu, L.; Hall, D. J.; Qin, Z.; Anglin, E.; Joo, J.; Mooney, D. J.; Howell, S. B.; Sailor, M. J. In Vivo Time-Gated Fluorescence Imaging With Biodegradable Luminescent Porous Silicon Nanoparticles. Nat. Commun. 2013, 4, 2326.

(8) Park, J.-H.; von Maltzahn, G.; Ong, L. L.; Centrone, A.; Hatton, T. A.; Ruoslahti, E.; Bhatia, S. N.; Sailor, M. J. Cooperative Nanoparticles for Tumor Detection And Photothermally Triggered Drug Delivery. Adv. Mater. 2010, 22, 880-885.

(9) Jane, A.; Dronov, R.; Hodges, A.; Voelcker, N. H. Porous Silicon Biosensors on the Advance. Trends Biotechnol. 2009, 27, 230-239.

(10) Sailor, M. J. Porous Silicon in Practice: Preparation, Characterization and Applications; John Wiley \& Sons: Weinheim, 2012.

(11) Föll, H.; Christophersen, M.; Carstensen, J.; Hasse, G. Formation and Application of Porous Silicon. Mater. Sci. Eng., $R$ 2002, 39, 93-141.

(12) Janshoff, A.; Dancil, K.-P. S.; Steinem, C.; Greiner, D. P.; Lin, V. S. Y.; Gurtner, C.; Motesharei, K.; Sailor, M. J.; Ghadiri, M. R. Macroporous p-type Silicon Fabry-Perot Layers. Fabrication, Characterization, and Applications in Biosensing. J. Am. Chem. Soc. 1998, 120, 12108-12116.

(13) Ruminski, A. M.; Barillaro, G.; Secret, E.; Huang, W. D.; Potocny, A.; Carion, U.; Wertans, C.; Sailor, M. J. Topological Control of Porous Silicon Photonic Crystals by Microcontact Printing. Adv. Opt. Mater. 2013, 1, 510-516.

(14) Maniya, N. H.; Patel, S. R.; Murthy, Z. V. P. Simulation and Fabrication Study of Porous Silicon Photonic Crystal. Optik 2014, 125, 828-831.

(15) Pavesi, L.; Dubos, P. Random Porous Silicon Multilayers: Application to Distributed Bragg Reflectors and Interferential Fabry Pérot Filters. Semicond. Sci. Technol. 1997, 12, 570.

(16) Thuy Chi, D.; Huy, B.; Thuy Van, N.; The Anh, N.; Thanh Hai, N.; Van Hoi, P. A. Microcavity Based on a Porous Silicon Multilayer. Adv. Nat. Sci.: Nanosci. Nanotechnol. 2011, 2, 035001.

(17) Pavesi, L.; Panzarini, G.; Andreani, L. C. All-Porous SiliconCoupled Microcavities: Experiment Versus Theory. Phys. Rev. B: Condens. Matter Mater. Phys. 1998, 58, 15794-15800.

(18) Salem, M. S.; Sailor, M. J.; Sakka, T.; Ogata, Y. H. Electrochemical Preparation of a Rugate Filter in Silicon and its Deviation from the Ideal Structure. J. Appl. Phys. 2007, 101, 063503.

(19) Robbiano, V.; Di Stasio, F.; Surdo, S.; Mian, S.; Barillaro, G.; Cacialli, F.. Hybrid-Organic Photonic Structures for Light Emission Modification. In Organic and Hybrid Photonic Crystals; Comoretto, D., Ed.; Springer International Publishing: Cham, 2015; pp 339-358.

(20) Rong, G.; Najmaie, A.; Sipe, J. E.; Weiss, S. M. Nanoscale Porous Silicon Waveguide for Label-Free DNA Sensing. Biosens. Biosens. Bioelectron. 2008, 23, 1572-1576.

(21) Krueger, N. A.; Holsteen, A. L.; Kang, S. K.; Ocier, C. R.; Zhou, W.; Mensing, G.; Rogers, J. A.; Brongersma, M. L.; Braun, P. V. Porous Silicon Gradient Refractive Index Micro-Optics. Nano Lett. 2016, 16, 7402-7407.

(22) Jenie, S. N. A.; Pace, S.; Sciacca, B.; Brooks, R. D.; Plush, S. E.; Voelcker, N. H. Lanthanide Luminescence Enhancements in Porous Silicon Resonant Microcavities. ACS Appl. Mater. Interfaces 2014, 6, 12012-12021.

(23) Ning, H.; Krueger, N. A.; Sheng, X.; Keurn, H.; Zhang, C.; Choquette, K. D.; Li, X. L.; Kim, S.; Rogers, J. A.; Braun, P. V. Transfer-Printing of Tunable Porous Silicon Microcavities with Embedded Emitters. ACS Photonics 2014, 1, 1144-1150.

(24) Wan, Y.; Krueger, N. A.; Ocier, C. R.; Su, P.; Braun, P. V.; Cunningham, B. T. Resonant Mode Engineering of Photonic Crystal Sensors Clad with Ultralow Refractive Index Porous Silicon Dioxide. Adv. Opt. Mater. 2017, 5, 1700605.

(25) Ocier, C. R.; Krueger, N. A.; Zhou, W. J.; Braun, P. V. Tunable Visibly Transparent Optics Derived from Porous Silicon. ACS Photonics 2017, 4, 909-914.
(26) Ryu, G.; Xia, R.; Bradley, D. D. C. Optical Gain Characteristics of $\beta$-phase Poly(9,9-dioctylfluorene). J. Phys.: Condens. Matter 2007, $19,056205$.

(27) Heliotis, G.; Xia, R.; Turnbull, G. A.; Andrew, P.; Barnes, W. L.; Samuel, D. W.; Bradley, D. D. C. Emission Characteristics and Performance Comparison of Polyfluorene Lasers with One- And TwoDimensional Distributed Feedback. Adv. Funct. Mater. 2004, 14, 9197.

(28) O'Carroll, D.; Lieberwirth, I.; Redmond, G. Microcavity Effects and Optically Pumped Lasing in Single Conjugated Polymer Nanowires. Nat. Nanotechnol. 2007, 2, 180-184.

(29) Edward, P. D.; Holm, R. T. Handbook of Optical Constants of Solids; Academic Press: New York, 1998.

(30) Stavrinou, P. N.; Ryu, G.; Campoy-Quiles, M.; Bradley, D. D. C. The Change in refractive Index of poly(9,9-dioctylfluorene) Due to the Adoption of the $\beta$-phase Chain Conformation. J. Phys.: Condens. Matter 2007, 19, 056205.

(31) Chen, S. H.; Su, A. C.; Su, C. H.; Chen, S. A. Crystalline Forms and Emission Behavior of Poly(9,9-di-n-octyl-2,7-fluorene). Macromolecules 2005, 38, 379-385.

(32) Kavokin, A. V.; Baumberg, J. J.; Malpuech, G.; Laussy, F. P. Microcavities; Oxford University Press: Oxford, 2017.

(33) Di Stasio, F.; Berti, L.; McDonnell, S. O.; Robbiano, V.; Anderson, H. L.; Comoretto, D.; Cacialli, F. Fluorescent Polystyrene Photonic Crystals Self-Assembled with Water-Soluble Conjugated Polyrotaxanes. APL Mater. 2013, 1, 042116.

(34) Cornil, J.; Beljonne, D.; Calbert, J. P.; Brédas, J. L. Interchain Interactions in Organic $\pi$-Conjugated Materials: Impact on Electronic Structure, Optical Response, and Charge Transport. Adv. Mater. 2001, 13, 1053-1067.

(35) Spano, F. C. The Spectral Signatures of Frenkel Polarons in Hand J-Aggregates. Acc. Chem. Res. 2010, 43, 429-439.

(36) Cornil, J.; dos Santos, D. A.; Crispin, X.; Silbey, R.; Brédas, J. L. Influence of Interchain Interactions on the Absorption and Luminescence of Conjugated Oligomers and Polymers: a QuantumChemical Characterization. J. Am. Chem. Soc. 1998, 120, 1289-1299.

(37) Brédas, J.-L.; Cornil, J.; Heeger, A. J. The Exciton Binding Energy in Luminescent Conjugated Polymers. Adv. Mater. 1996, 8, $447-452$.

(38) Gierschner, J.; Ehni, M.; Egelhaaf, H.-J.; Milián Medina, B.; Beljonne, D.; Benmansour, H.; Bazan, G. C. Solid-State Optical Properties of Linear Polyconjugated Molecules: $\pi$-Stack Contra Herringbone. J. Chem. Phys. 2005, 123, 144914.

(39) P Petrozza, A.; Brovelli, S.; Michels, J. J.; Anderson, H. L.; Friend, R. H.; Silva, C.; Cacialli, F. Control of Rapid Formation of Interchain Excited States in Sugar-Threaded Supramolecular Wires. Adv. Mater. 2008, 20, 3218-3223.

(40) Gérard, J. M.; Sermage, B.; Gayral, B.; Legrand, B.; Costard, E.; Thierry-Mieg, V. Enhanced Spontaneous Emission by Quantum Boxes in a Monolithic Optical Microcavity. Phys. Rev. Lett. 1998, 81, 11101113.

(41) Adawi, A. M.; Cadby, A.; Connolly, L. G.; Hung, W. C.; Dean, R.; Tahraoui, A.; Fox, A. M.; Cullis, A. G.; Sanvitto, D.; Skolnick, M. S.; Lidzey, D. G. Spontaneous Emission Control in Micropillar Cavities Containing a Fluorescent Molecular Dye. Adv. Mater. 2006, $18,742-747$.

(42) Wang, J.; Cao, R.; Da, P.; Wang, Y.; Hu, T.; Wu, L.; Lu, J.; Shen, X.; Xu, F.; Zheng, G.; Chen, Z. Purcell Effect in an Organic-Inorganic Halide Perovskite Semiconductor Microcavity System. Appl. Phys. Lett. 2016, 108, 022103 .

(43) Yamamoto, Y.; Machida, S.; Björk, G. Micro-Cavity Semiconductor Lasers with Controlled Spontaneous Emission. Opt. Quantum Electron. 1992, 24, S215-S243.

(44) Noda, S.; Fujita, M.; Asano, T. Spontaneous-Emission Control by Photonic Crystals and Nanocavities. Nat. Photonics 2007, 1, 449458 .

(45) Purcell, E. M. Spontaneous Emission Probabilities at Radio Frequencies. Phys. Rev. 1946, 69, 681. 
(46) Tessler, N.; Denton, G. J.; Friend, R. H. Lasing from Conjugated-Polymer Microcavities. Nature 1996, 382, 695-697.

(47) Persano, L.; Del Carro, P.; Mele, E.; Cingolani, R.; Pisignano, D.; Zavelani-Rossi, M.; Longhi, S.; Lanzani, G. Monolithic Polymer Microcavity Lasers with On-Top Evaporated Dielectric Mirrors. Appl. Phys. Lett. 2006, 88, 121110.

(48) Svelto, O.; Hanna, D. C. Principles of Lasers; Springer: New York, 1998.

(49) Samuel, I. D. W.; Turnbull, G. A. Organic Semiconductor Lasers. Chem. Rev. 2007, 107, 1272-1295.

(50) Ruminski, A. M.; Barillaro, G.; Chaffin, C.; Sailor, M. J. Internally Referenced Remote Sensors for $\mathrm{HF}$ and $\mathrm{Cl}_{2}$ Using Reactive Porous Silicon Photonic Crystals. Adv. Funct. Mater. 2011, 21, 15111525. 